\title{
Highly diastereoselective templated complexation of dipyrromethenes
}

\author{
Adeeb Al-Sheikh-Ali, Kyle S. Cameron, T. Stanley Cameron, ${ }^{\dagger}$ Katherine N. Robertson ${ }^{\dagger}$ and \\ Alison Thompson* \\ Department of Chemistry, Dalhousie University, Halifax, Nova Scotia, B3H 4J3, Canada \\ alison.thompson@dal.ca
}

\section{Table of Contents}

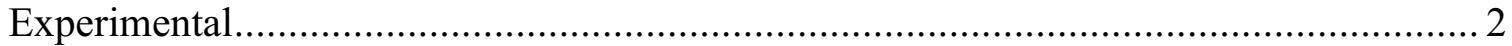

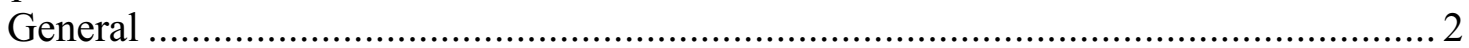

Benzyl 4-ethanoic acid-3,5-dimethyl-1H-pyrrole-2-carboxylate (2)........................... 2

Bis[benzyl 4,4' -[(R)-binolethanoate-3,5-dimethyl]-1H-pyrrole-2-carboxylate] ............ 3

3,3'-[(R)-Binolethanoate]bis[2,4,dimethyl-5-[(4-ethyl-3,5-dimethyl-1H-pyrrol-2ylidene)methyl]]-, 1H Pyrrole Dihydrobromide (4) ............................................ 3

Zinc, Bis[m-[[3,3'-[(R)-binolethanoate]bis[2,4,dimethyl-5-[(4-ethyl-3,5-dimethyl-1Hpyrrol-2-ylidene)methyl]-1H-pyrrolato-kN]](2-)]]di- (6) ................................... 4

Bis[benzyl 4,4'-[dimethyl- $(R, R)$-tartrylethanoate-3,5-dimethyl]-1H-pyrrole-2-

carboxylate]

3,3'-[Dimethyl- $(R, R)$-tartrylethanoate]bis[2,4,dimethyl-5-[(4-ethyl-3,5-dimethyl-1H-

pyrrol-2-ylidene)methyl]]-, 1H Pyrrole Dihydrobromide (5)

Zinc, Bis[m-[[3,3'-[dimethyl- $(R, R)$-tartrylethanoate]bis[2,4,dimethyl-5-[(4-ethyl-3,5-

dimethyl-1H-pyrrol-2-ylidene)methyl]-1H-pyrrolato-kN]](2-)]]di- (7) ..................... 5

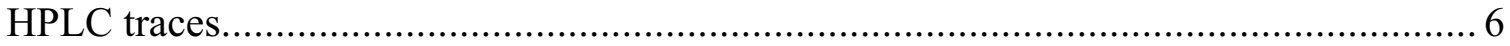

HPLC trace of complexation reaction containing binol zinc complex 6 ....................6 6

HPLC trace of complexation reaction containing tartrate zinc complex 7 ................. 6

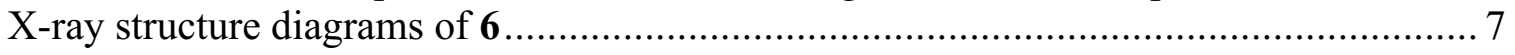

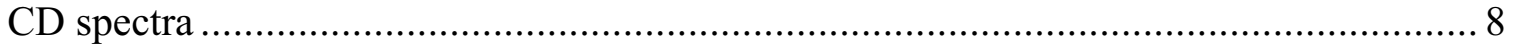

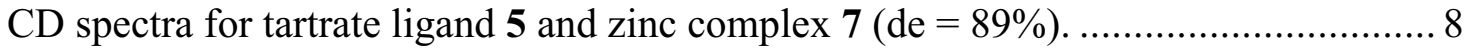

${ }^{13} \mathrm{C}$ NMR spectra..................................................................................... 9

${ }^{13} \mathrm{C}$ spectrum of bis[benzyl 4,4'-[(R)-binolethanoate-3,5-dimethyl]-1H-pyrrole-2-

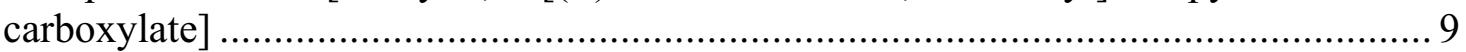

${ }^{13} \mathrm{C}$ spectrum of 3,3'-[(R)-binolethanoate]bis[2,4, dimethyl-5-[(4-ethyl-3,5-dimethyl-

1H-pyrrol-2-ylidene)methyl]-, $1 \mathrm{H}$ pyrrole dihydrobromide (4) .............................. 9

${ }^{13} \mathrm{C}$ spectrum of zinc, bis[m-[[3,3' - [(R)-binolethanoate]bis[2,4, dimethyl-5-[(4-ethyl-

3,5-dimethyl-1H-pyrrol-2-ylidene)methyl]-1H-pyrrolato-kN]](2-)]]di- (6) ............... 10

${ }^{13} \mathrm{C}$ spectrum of bis[benzyl 4,4'-[dimethyl- $(R, R)$-tartrylethanoate-3,5-dimethyl]-1H-

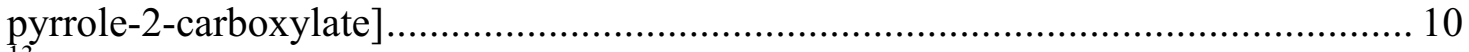

${ }^{13} \mathrm{C}$ spectrum of 3,3' -[dimethyl- $(R, R)$-tartrylethanoate]bis[2,4, dimethyl-5-[(4-ethyl-3,5-

dimethyl-1H-pyrrol-2-ylidene)methyl]-, $1 \mathrm{H}$ pyrrole dihydrobromide (5) ................... 11

${ }^{13} \mathrm{C}$ spectrum of zinc, bis[m-[[3,3'-[dimethyl- $(R, R)$-tartrylethanoate]bis[2,4,dimethyl-5[(4-ethyl-3,5-dimethyl-1H-pyrrol-2-ylidene)methyl]-1H-pyrrolato-kN]](2-)]]di- (7) . 11 


\section{Highly diastereoselective templated complexation of dipyrromethenes}

\section{Experimental}

\section{General}

All nuclear magnetic resonance experiments were conducted using either a Bruker AC250 or a Bruker AV-500 spectrometer, as indicated. All ${ }^{1} \mathrm{H}$ NMR chemical shifts are reported using tetramethylsilane as an internal reference at $0.00 \mathrm{ppm}$, with coupling constants $(J)$ being reported in $\mathrm{Hz}$. All ${ }^{13} \mathrm{C}$ NMR chemical shifts are reported relative to solvents peaks as internal references: $\mathrm{CDCl}_{3}(77.23 \mathrm{ppm})$ and acetone- $d_{6}(29.92 \mathrm{ppm})$. All HPLC analyses were conducted using a Varian ProStar 330 system. Analytical chiral HPLC was performed using a CHIRALPAK ${ }^{\circledR}$ IA column $5 \mu \mathrm{m} 250$ x $4.6 \mathrm{~mm}$ (L x I.D.), eluting with $\mathrm{CH}_{3} \mathrm{OH}: \mathrm{CHCl}_{3}$ (98:2) with flow rate of $\left(0.5 \mathrm{mLmin}^{-1}\right)$ and monitoring at $\lambda=$ $489 \mathrm{~nm}$. Mass spectra were obtained using CEC 21-110B sector (EI) and Thermo Finnigan LCQ Duo ion trap (ESI) instruments. All CD spectra were obtained using a Jasco J-810 Spectropolarimeter with a $2 \mathrm{~mm}$ cell and $\mathrm{CH}_{3} \mathrm{OH}: \mathrm{CHCl}_{3}(98: 2)$ as solvent. All UV-visible analyses were performed using a Varian (CARY-100 Bio) spectrophotometer with $10 \mathrm{~mm}$ cell and $\mathrm{CH}_{3} \mathrm{OH}: \mathrm{CHCl}_{3}(98: 2)$ as solvent. All melting points are determined through use of a Fisher-John melting point apparatus, and are reported uncorrected. Chromatography was performed using 230-400 mesh Silicycle ultra pure silica. Unless otherwise stated, chemicals were received from suppliers and used without further purification. Dry, deoxygenated tetrahydrofuran was obtained from an Innovative Technology solvent purification system. Benzyl 4-(methoxycarbonylmethyl)3,5-dimethyl-pyrrole-2-carboxylate (1) was prepared according to Paine III, J.B.; Dolphin, D. J. Org. Chem. 1985, 50, 5598-5604. Benzyl 4-(methoxycarbonylethyl)-3,5dimethyl-pyrrole-2-carboxylate was prepared according to Johnson, A.W. ; Markham, E.; Price, R.; Shaw, K.B. J. Chem. Soc. 1958, 4254-4257. 2-Formyl-4-ethyl-3,5dimethylpyrrole (3) was prepared according to Clezy, P.S.; Fookes, C.J.R.; Prashar, J.K. Aust. J. Chem. 1989, 42, 775-86.

Benzyl 4-ethanoic acid-3,5-dimethyl-1H-pyrrole-2-carboxylate (2)

A solution of lithium hydroxide monohydrate $(2.0 \mathrm{~g}, 47 \mathrm{mmol})$ in a $1: 1(\mathrm{v} / \mathrm{v})$ tetrahydrofuran:water solution $(40 \mathrm{~mL})$ was added to a solution of benzyl 4(methoxycarbonylmethyl)-3,5-dimethylpyrrole-2-carboxylate (1) (14 g, 47 mmol) dissolved in a 1:1 (v/v) tetrahydrofuran:water solution $(100 \mathrm{~mL})$. The mixture was stirred 


\section{Highly diastereoselective templated complexation of dipyrromethenes}

at room temperature for 24 hours before being washed with ethyl acetate $(2 \times 30 \mathrm{~mL})$. The aqueous layer was acidified with a $5 \%(\mathrm{~m} / \mathrm{v})$ aqueous solution of citric acid, resulting in the precipitation of a white solid. The product was collected, by suction filtration, as a white powder (12.2 g, 91\%): mp 193-194 ${ }^{\circ} \mathrm{C} ; \delta_{\mathrm{H}}\left(250 \mathrm{MHz}\right.$, acetone- $\left.\mathrm{d}_{6}\right) 10.37(1 \mathrm{H}, \mathrm{br} \mathrm{s}$, $\mathrm{NH}), 7.30-7.44(5 \mathrm{H}, \mathrm{m}, \mathrm{ArH}), 5.26\left(2 \mathrm{H}, \mathrm{s}, \mathrm{CH}_{2} \mathrm{Ph}\right), 3.38\left(2 \mathrm{H}, \mathrm{s}, \mathrm{CH}_{2} \mathrm{CO}\right), 2.29(3 \mathrm{H}, \mathrm{s}$, $\left.\mathrm{CH}_{3}\right), 2.24\left(3 \mathrm{H}, \mathrm{s}, \mathrm{CH}_{3}\right) ; \delta_{\mathrm{C}}\left(126 \mathrm{MHz}\right.$, acetone-d $\left.\mathrm{d}_{6}\right) 173.1,161.6,138.3,132.5,129.3$, 128.9, 128.7, 128.6, 117.4, 115.9, 65.5, 30.2, 11.3, 10.9; m/z (EI) $287\left(\mathrm{M}^{+}, 7 \%\right), 91$ $\left(\mathrm{PhCH}_{2}{ }^{+}, 100\right)$; found $\mathrm{M}^{+} 287.1160, \mathrm{C}_{16} \mathrm{H}_{17} \mathrm{NO}_{4}$ requires 287.1157 .

\section{Bis[benzyl 4,4'-[(R)-binolethanoate-3,5-dimethyl]-1H-pyrrole-2-carboxylate]}

To a stirring solution of carboxylic acid (2) $(10.0 \mathrm{~g}, 34.8 \mathrm{mmol})$ in dry THF $(100 \mathrm{~mL})$ was added 4-( $N, N$-dimethylaminopyridine) (DMAP) $(430 \mathrm{mg}, 3.50 \mathrm{mmol})$, followed by $(R)$ binol (4.49 g, $15.7 \mathrm{mmol}$.). The solution was cooled to $0^{\circ} \mathrm{C}$, at which point dicyclohexylcarbodiimide (DCC) $(7.54 \mathrm{~g}, 36.6 \mathrm{mmol})$ was added and the solution was stirred at room temperature for 24 hours. The reaction mixture was filtered to remove the solid dicyclohexylurea and then the solvent was removed in vacuo. The resultant crude product was purified by column chromatography on silica gel, eluting with $20 \%$ ethyl acetate in hexane. Removal of the solvent in vacuo, then trituration of the solid with chloroform $(1 \mathrm{~mL})$ gave a white powder $(12.9 \mathrm{mg}, 90 \%)$ : $\mathrm{mp} 93-96^{\circ} \mathrm{C}$; UV-vis $\lambda_{\max } / \mathrm{nm}$ 226 (ع 86,000); $[\Theta]_{228}-3.85 \times 10^{5} \mathrm{deg} \mathrm{cm}^{2} \mathrm{dmol}^{-1} ; \delta_{\mathrm{H}}\left(500 \mathrm{MHz}, \mathrm{CDCl}_{3}\right) 8.38(1 \mathrm{H}, \mathrm{s}, \mathrm{NH})$, $7.75(1 \mathrm{H}, \mathrm{d}, J 9, \operatorname{Ar} H), 7.74(1 \mathrm{H}, \mathrm{d}, J 8, \operatorname{Ar} H), 7.53-2.57(2 \mathrm{H}, \mathrm{m}, \operatorname{Ar} H), 7.48(1 \mathrm{H}, \mathrm{d}, J 8$, $\operatorname{Ar} H), 7.45(1 \mathrm{H}, \mathrm{d}, J$ 8, $\operatorname{Ar} H), 7.38-7.43(1 \mathrm{H}, \mathrm{m} \mathrm{Ar} H), 7.28-7.35(1 \mathrm{H}, \mathrm{m}, \operatorname{Ar} H), 7.24(1 \mathrm{H}$, d, $J$ 9, $\operatorname{Ar} H), 7.16-7.23(1 \mathrm{H}, \mathrm{m}, \operatorname{ArH}), 7.05(1 \mathrm{H}, \mathrm{d}, J 9, \operatorname{Ar} H), 5.39\left(4 \mathrm{H}, \mathrm{s}, \operatorname{ArCH}_{2}\right), 3.18$ $\left(4 \mathrm{H}, \mathrm{ABq}, J 15, \mathrm{CH}_{2} \mathrm{CO}\right), 1.91\left(6 \mathrm{H}, \mathrm{s}, \mathrm{CH}_{3}\right), 1.73\left(6 \mathrm{H}, \mathrm{s}, \mathrm{CH}_{3}\right) ; \delta_{\mathrm{C}}\left(126 \mathrm{MHz}, \mathrm{CDCl}_{3}\right)$ 169.5, 161.4, 146.7, 137.0, 133.3, 131.6, 131.4, 129.6, 129.0, 128.7, 128.6, 128.1, 126.7, $126.1,125.8,123.5,121.7,116.8,113.4,65.8,30.3,11.2,10.5 ; \mathrm{m} / \mathrm{z}(\mathrm{ESI}) 847.3(\mathrm{M}+$ $\mathrm{Na})^{+}$.

\section{3,3'-I(R)-Binolethanoate]bis[2,4,dimethyl-5-[(4-ethyl-3,5-dimethyl-1H-pyrrol-2-} ylidene)methyll]-, $1 \mathrm{H}$ Pyrrole Dihydrobromide (4)

To a suspension of bis[benzyl 4,4'-[(R)-binolethanoate-3,5-dimethyl]-1H-pyrrole-2carboxylate] (330 mg, $0.4 \mathrm{mmol})$ and 10\% Pd on activated carbon $(20 \mathrm{mg}$ ) was added THF $(10 \mathrm{~mL})$ under nitrogen. The reaction mixture was exposed to hydrogen at $1 \mathrm{~atm}$. 


\section{Highly diastereoselective templated complexation of dipyrromethenes}

with continuous stirring for $15 \mathrm{~h}$, after which time the starting material was completely consumed according to TLC. The reaction mixture was filtered through Celite ${ }^{\circledR}$ which was then washed with THF $(5 \mathrm{~mL})$ and $\mathrm{MeOH}(5 \mathrm{~mL})$. To the filtrate, 4-ethyl-2-formyl3,5-dimethyl-pyrrole (3) (121 mg, $0.80 \mathrm{mmol})$ and three drops of concentrated $\mathrm{HBr}$ $(48 \%)$ were added, and the reaction mixture was stirred at $40^{\circ} \mathrm{C}$ for $1.5 \mathrm{~h}$, after which time the aldehyde was completely consumed, according to TLC. Removal of solvents in vacuo, followed by addition of $\mathrm{CH}_{2} \mathrm{Cl}_{2}(40 \mathrm{~mL})$, drying of the solution over $\mathrm{MgSO}_{4}$, and removal of the organic solvent in vacuo gave the crude product. Trituration of the crude solid with ether gave a reddish brown powder $(348 \mathrm{mg}, 88 \%)$ : $\mathrm{mp}(\mathrm{dec})>162^{\circ} \mathrm{C}$; $\mathrm{UV}$-vis

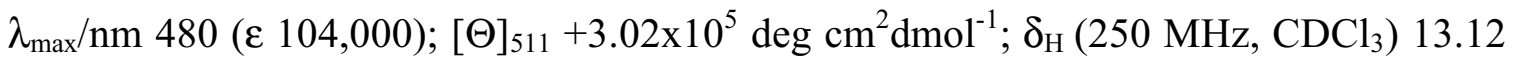
$(1 \mathrm{H}, \mathrm{s}, \mathrm{NH}), 12.66(1 \mathrm{H}, \mathrm{s}, \mathrm{NH}), 7.83(1 \mathrm{H}, \mathrm{d}, J 10, \operatorname{Ar} H), 7.68(1 \mathrm{H}, \mathrm{d}, J$ 8, $\operatorname{ArH}), 7.40(1 \mathrm{H}$, $\mathrm{d}, J 8, \operatorname{Ar} H), 7.32-7.38(1 \mathrm{H}, \mathrm{m}, \operatorname{Ar} H), 7.13-7.19(1 \mathrm{H}, \mathrm{m}, \operatorname{Ar} H), 7.00(1 \mathrm{H}, \mathrm{d}, J 8, \operatorname{Ar} H)$, $6.80(1 \mathrm{H}, \mathrm{s}, \mathrm{CH}=\mathrm{C}), 3.25\left(2 \mathrm{H}, \mathrm{ABq}, J 15, \mathrm{CH}_{2} \mathrm{CO}\right), 2.77\left(3 \mathrm{H}, \mathrm{s}, \mathrm{CH}_{3}\right), 2.49(2 \mathrm{H}, \mathrm{q}, J 8$, $\left.\mathrm{CH}_{2} \mathrm{CH}_{3}\right), 2.35\left(3 \mathrm{H}, \mathrm{s}, \mathrm{CH}_{3}\right), 2.25(3 \mathrm{H}, \mathrm{s}, \mathrm{CH}), 1.75(3 \mathrm{H}, \mathrm{s}, \mathrm{CH}), 1.15(3 \mathrm{H}, \mathrm{t}, J 8$, $\left.\mathrm{CH}_{2} \mathrm{CH}_{3}\right) ; \delta_{\mathrm{C}}\left(126 \mathrm{MHz}, \mathrm{CDCl}_{3}\right) 167.9,155.3,153.2,146.3,142.8,142.0,133.0,131.5$, $131.3,130.1,128.2,126.8,126.5,126.2,125.9,125.5,123.1,121.7,119.5,119.3,30.1$, $17.5,14.6,13.1,12.3,10.2,9.8 ; \mathrm{m} / z(\mathrm{ESI}+) 823.3\left(\mathrm{M}^{+}-2 \mathrm{HBr}\right)$.

\section{Zinc, Bis/m-[[3,3'-[(R)-binolethanoate]bis[2,4,dimethyl-5-[(4-ethyl-3,5-dimethyl-1H- pyrrol-2-ylidene)methyl]-1H-pyrrolato-kN]J(2-)]Jdi- (6)}

To a solution of bis(dipyrromethene) hydrobromide salt 4 (148 mg, $0.15 \mathrm{mmol})$ in $\mathrm{CHCl}_{3}$ $(3 \mathrm{~mL})$ was added a solution of $\mathrm{Zn}(\mathrm{OAc})_{2} \cdot 2 \mathrm{H}_{2} \mathrm{O}(308 \mathrm{mg}, 1.4 \mathrm{mmol})$ and $\mathrm{NaOAc} .3 \mathrm{H}_{2} \mathrm{O}$ (190 mg, $1.4 \mathrm{mmol})$ in $\mathrm{CH}_{3} \mathrm{OH}(3 \mathrm{~mL})$. The reaction mixture was stirred at $40^{\circ} \mathrm{C}$ for 2.5 h. Removal of solvents in vacuo, followed by dissolution of the residue in $\mathrm{CH}_{2} \mathrm{Cl}_{2}(20$ $\mathrm{mL})$, washing with $\mathrm{H}_{2} \mathrm{O}(2 \times 10 \mathrm{~mL})$, then drying the organic fraction over $\mathrm{MgSO}_{4}$, removal of the solvent in vacuo gave the crude product as a film. Trituration of the crude solid with hexane gave a reddish brown powder as a single diastereoisomer according to ${ }^{1}$ HNMR spectroscopy and $>99 \%$ according to chiral HPLC (121 mg, 91\%): $\mathrm{mp}(\mathrm{dec})>$ $212^{\circ} \mathrm{C}$; UV-vis $\lambda_{\max } / \mathrm{nm} 473$ ( $\varepsilon$ 123,000); $[\Theta]_{510}+4.75 \times 10^{6} \mathrm{deg} \mathrm{cm}^{2} \mathrm{dmol}^{-1} ; \delta_{\mathrm{H}}(250 \mathrm{MHz}$, $\left.\mathrm{CDCl}_{3}\right)$ 8.85-7.93 (2H, m, ArH), 7.44-7.40 (1H, m, $\left.\mathrm{ArH}\right), 7.28-7.24$ (2H, m, ArH), 7.08 $(1 \mathrm{H}, \mathrm{d}, J 8, \mathrm{Ar} H), 7.06(1 \mathrm{H}, \mathrm{s}, \mathrm{CH}=\mathrm{C}), 3.16(1 \mathrm{H}, \mathrm{d}, J 15, \mathrm{CH}(\mathrm{H}) \mathrm{CO}), 2.83(1 \mathrm{H}, \mathrm{d}, J 15$, $\mathrm{CH}(\mathrm{H}) \mathrm{CO}), 2.42\left(2 \mathrm{H}, \mathrm{q}, J 8, \mathrm{CH}_{2} \mathrm{CH}_{3}\right), 2.35\left(3 \mathrm{H}, \mathrm{s}, \mathrm{CH}_{3}\right), 2.30(3 \mathrm{H}, \mathrm{s}, \mathrm{CH}), 1.98(3 \mathrm{H}, \mathrm{s}$, 


\section{Highly diastereoselective templated complexation of dipyrromethenes}

$\left.\mathrm{CH}_{3}\right), 1.48\left(3 \mathrm{H}, \mathrm{s}, \mathrm{CH}_{3}\right), 1.05\left(3 \mathrm{H}, \mathrm{t}, J\right.$ 8, $\left.\mathrm{CH}_{2} \mathrm{CH}_{3}\right) ; \delta_{\mathrm{C}}\left(126 \mathrm{MHz}, \mathrm{CDCl}_{3}\right)$ 169.6, 158.6, 155.4, 147.0, 137.9, 136.8, 136.3, 134.7, 133.2, 131.4, 130.8, 129.4, 127.9, 126.9, 126.0, $125.7,123.3,121.4,121.1,119.2,31.3,17.9,14.9,14.8,13.8,10.0,9.9 ; \mathrm{m} / z(\mathrm{ESI}+)$ $884.3\left(\mathrm{M}^{+}\right)$.

Bis[benzyl 4,4'-[dimethyl-(R,R)-tartrylethanoate-3,5-dimethyl]-1H-pyrrole-2carboxylate]

Following the procedure as for bis[benzyl 4,4'-[(R)-binolethanoate-3,5-dimethyl]- $1 H$ pyrrole-2-carboxylate], carboxylic acid $2(540 \mathrm{mg}, 1.79 \mathrm{mmol})$ in dry THF $(6 \mathrm{~mL})$ was reacted with DMAP (22 mg, $0.179 \mathrm{mmol})$, dimethyl $(R, R)$-tartrate (144 mg, $0.81 \mathrm{mmol})$ and DCC (388 mg, $1.88 \mathrm{mmol})$. Work-up as before gave a white solid (1.05 g, 82\%): $\mathrm{mp}$ 83-85 $\mathrm{C}$; UV-vis $\lambda_{\max } / \mathrm{nm} 280\left(\varepsilon\right.$ 40,000); $\delta_{\mathrm{H}}\left(500 \mathrm{MHz}, \mathrm{CDCl}_{3}\right) 8.95(1 \mathrm{H}, \mathrm{s}, \mathrm{NH}), 7.30-$ $7.40(5 \mathrm{H}, \mathrm{m}, \mathrm{Ar} H), 5.62(1 \mathrm{H}, \mathrm{s}, \mathrm{OCHCO}), 5.29\left(2 \mathrm{H}, \mathrm{s}, \mathrm{ArCH}_{2}\right), 3.55\left(3 \mathrm{H}, \mathrm{s}, \mathrm{OCH}_{3}\right), 3.44$ $\left(2 \mathrm{H}, \mathrm{s}, \mathrm{CH}_{2} \mathrm{CO}\right), 2.27\left(3 \mathrm{H}, \mathrm{s}, \mathrm{CH}_{3}\right), 2.15\left(3 \mathrm{H}, \mathrm{s}, \mathrm{CH}_{3}\right) ; \delta_{\mathrm{C}}\left(126 \mathrm{MHz}, \mathrm{CDCl}_{3}\right) 170.5$, 166.3, 161.7, 136.8, 132.0, 128.9, 128.4, 117.1, 114.0, 71.2, 65.9, 53.0, 29.9, 11.6, 10.9; $\mathrm{m} / z(\mathrm{ESI}+) 739.2(\mathrm{M}+\mathrm{Na})^{+}$.

3,3'-[Dimethyl-(R,R)-tartrylethanoate]bis[2,4,dimethyl-5-[(4-ethyl-3,5-dimethyl-1Hpyrrol-2-ylidene)methyll]-, 1H Pyrrole Dihydrobromide (5)

Following the procedure as for $\mathbf{4}$, bis[benzyl 4,4'-[dimethyl- $(R, R)$-tartrylethanoate-3,5dimethyl]-1H-pyrrole-2-carboxylate $(287 \mathrm{mg}, 0.4 \mathrm{mmol}$ ) was hydrogenolyzed and the resulting dicarboxylic acid reacted with 4-ethyl-2-formyl-3,5-dimethyl-pyrrole (3) (121 $\mathrm{mg}, 0.80 \mathrm{mmol}$ ) and three drops of concentrated $\mathrm{HBr}(48 \%)$. Work-up as before gave an orange powder with a metallic green lustre $(831 \mathrm{mg}, 98 \%): \mathrm{mp}(\mathrm{dec})>186^{\circ} \mathrm{C}$; UV-vis $\lambda_{\max } / \mathrm{nm} 480\left(\varepsilon\right.$ 85,000); $[\Theta]_{508}+6.37 \times 10^{4} \mathrm{deg} \mathrm{cm}^{2} \mathrm{dmol}^{-1} ; \delta_{\mathrm{H}}\left(500 \mathrm{MHz}, \mathrm{CDCl}_{3}\right) 13.12$ $(1 \mathrm{H}, \mathrm{s}, \mathrm{N} H), 13.06(1 \mathrm{H}, \mathrm{s}, \mathrm{N} H), 7.13(1 \mathrm{H}, \mathrm{s}, \mathrm{CH}=\mathrm{C}), 5.66(1 \mathrm{H}, \mathrm{s}, \mathrm{OCHCO}), 3.67(3 \mathrm{H}, \mathrm{s}$, $\left.\mathrm{OCH}_{3}\right), 3.53\left(2 \mathrm{H}, \mathrm{s}, \mathrm{CH}_{2} \mathrm{CO}\right), 2.69\left(3 \mathrm{H}, \mathrm{s}, \mathrm{CH}_{3}\right), 2.65\left(3 \mathrm{H}, \mathrm{s}, \mathrm{CH}_{3}\right) 2.43(2 \mathrm{H}, \mathrm{q}, J 8$, $\left.\mathrm{CH}_{2} \mathrm{CH}_{3}\right), 2.31\left(6 \mathrm{H}, \mathrm{s}, 2 \mathrm{CH}_{3}\right), 1.07\left(3 \mathrm{H}, \mathrm{t}, J 8, \mathrm{CH}_{2} \mathrm{CH}_{3}\right) ; \delta_{\mathrm{C}}\left(126 \mathrm{MHz}, \mathrm{CDCl}_{3}\right) 168.8$, 165.8, 156.4, 152.6, 142.9, 142.4, 131.5, 126.9, 125.4, 119.6, 119.4, 71.1, 53.2, 29.6, $17.2,14.3,13.0,12.5,10.3,10.2 ; \mathrm{m} / z\left(\mathrm{ESI}^{+}\right) 715.3\left(\mathrm{M}^{+}-2 \mathrm{HBr}\right)$.

Zinc, Bis/m-[[3,3'-[dimethyl-(R,R)-tartrylethanoate]bis[2,4,dimethyl-5-[(4-ethyl-3,5dimethyl-1H-pyrrol-2-ylidene)methyl]-1H-pyrrolato-kN]J(2-)]]di- (7)

Following the procedure as for $\mathbf{6}$, bis(dipyrromethene) hydrobromide salt $5(132 \mathrm{mg}, 0.15$ mmol) was reacted with $\mathrm{Zn}(\mathrm{OAc})_{2} .2 \mathrm{H}_{2} \mathrm{O}(308 \mathrm{mg}, 1.4 \mathrm{mmol})$ and $\mathrm{NaOAc}^{2} 3 \mathrm{H}_{2} \mathrm{O}(190$ 


\section{Highly diastereoselective templated complexation of dipyrromethenes}

$\mathrm{mg}, 1.4 \mathrm{mmol}$ ). Work-up as before gave an orange powder with a metallic green lustre as a 9:1 mixture of diastereoisomers according to ${ }^{1} \mathrm{H}$ NMR spectroscopy and $89 \%$ de by chiral HPLC (106 mg, 91\%): $\mathrm{mp}(\mathrm{dec})>223^{\circ} \mathrm{C}$; UV-vis $\lambda_{\max } / \mathrm{nm} 504(\varepsilon 76,000) ;[\Theta]_{507}$ $+2.79 \times 10^{6} \mathrm{deg} \mathrm{cm}^{2} \mathrm{dmol}^{-1} ; \delta_{\mathrm{H}}\left(500 \mathrm{MHz}, \mathrm{CDCl}_{3}\right.$, major diastereoisomer) $7.05(1 \mathrm{H}, \mathrm{s}$, $\mathrm{C} H=\mathrm{C}), 5.59(1 \mathrm{H}, \mathrm{s}, \mathrm{OC} H \mathrm{CO}), 3.65\left(3 \mathrm{H}, \mathrm{s}, \mathrm{OCH}_{3}\right), 3.46(1 \mathrm{H}, \mathrm{d}, J 15, \mathrm{CH}(H) \mathrm{CO}), 3.38$ $(1 \mathrm{H}, \mathrm{d}, J 15, \mathrm{CH}(\mathrm{H}) \mathrm{CO}), 2.40\left(2 \mathrm{H}, \mathrm{q}, J 8, \mathrm{CH}_{2} \mathrm{CH}_{3}\right), 2.25(6 \mathrm{H}, \mathrm{s}, 2 \mathrm{CH}), 2.08(3 \mathrm{H}, \mathrm{s}$, $\left.\mathrm{CH}_{3}\right), 1.62\left(3 \mathrm{H}, \mathrm{s}, \mathrm{CH}_{3}\right), 1.06\left(3 \mathrm{H}, \mathrm{t}, J 8, \mathrm{CH}_{2} \mathrm{CH}_{3}\right) ; \delta_{\mathrm{C}}\left(126 \mathrm{MHz}, \mathrm{CDCl}_{3}\right.$, major diastereoisomer) 170.2, 166.1, 158.1, 156.2, 138.0, 137.6, 136.4, 135.2, 130.5, 121.6, $118.5,71.4,52.9,31.2,18.1,15.2,15.1,14.1,10.1,9.9 ; \mathrm{m} / z \mathrm{ESI}+799.3(\mathrm{M}+\mathrm{Na})^{+}$.

\section{HPLC traces}

HPLC trace of complexation reaction containing binol zinc complex 6

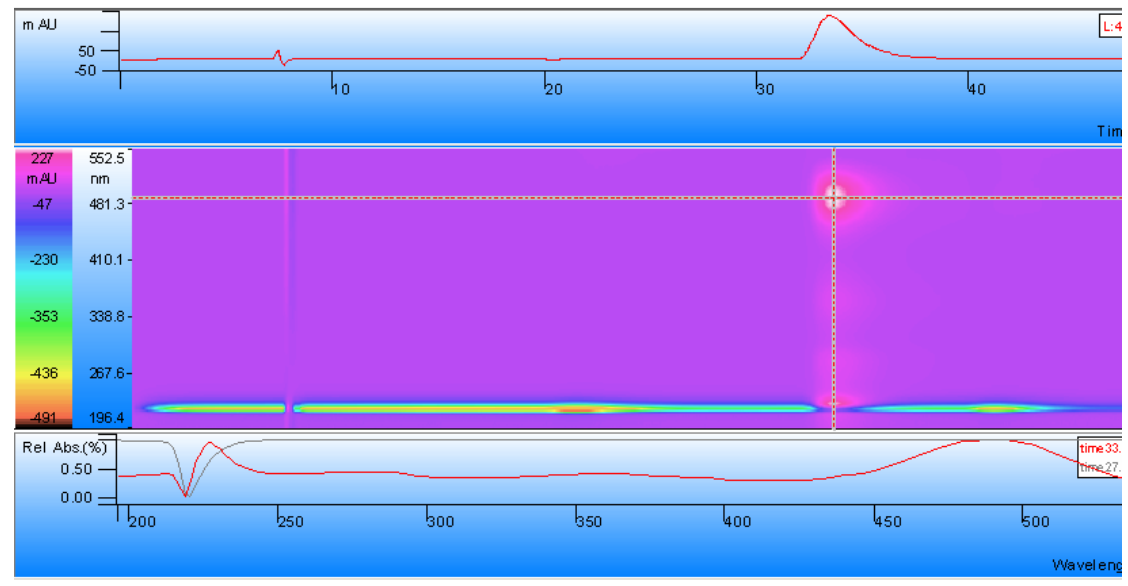

HPLC trace of complexation reaction containing tartrate zinc complex 7

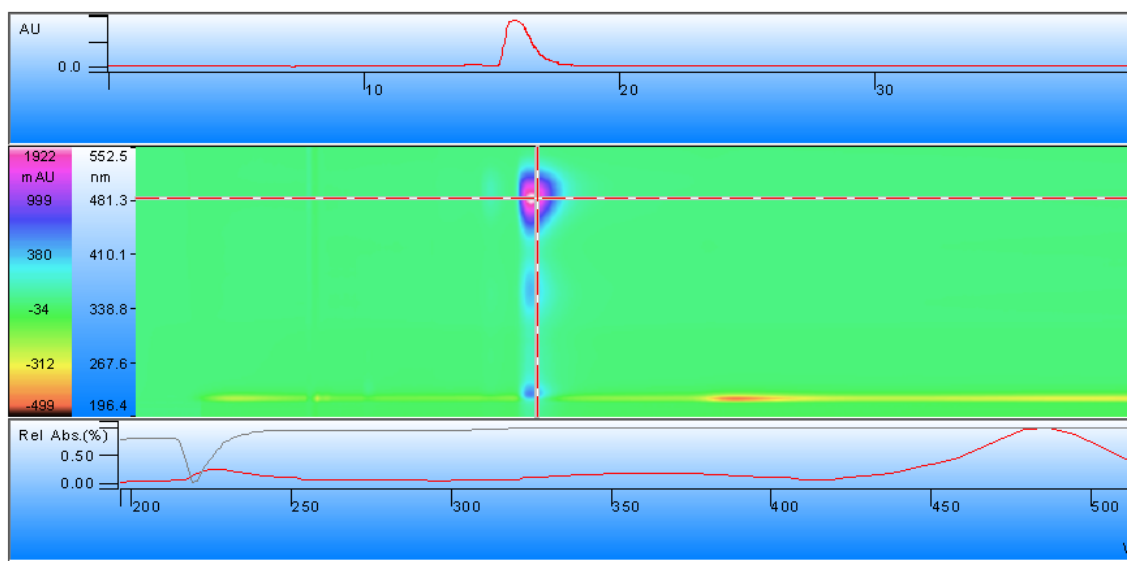

The peak at $14.2 \mathrm{~min}: 4.7 \%$.

The peak at $15.8 \mathrm{~min}: 93.3 \%$. 
Highly diastereoselective templated complexation of dipyrromethenes

\section{X-ray structure diagrams of 6}
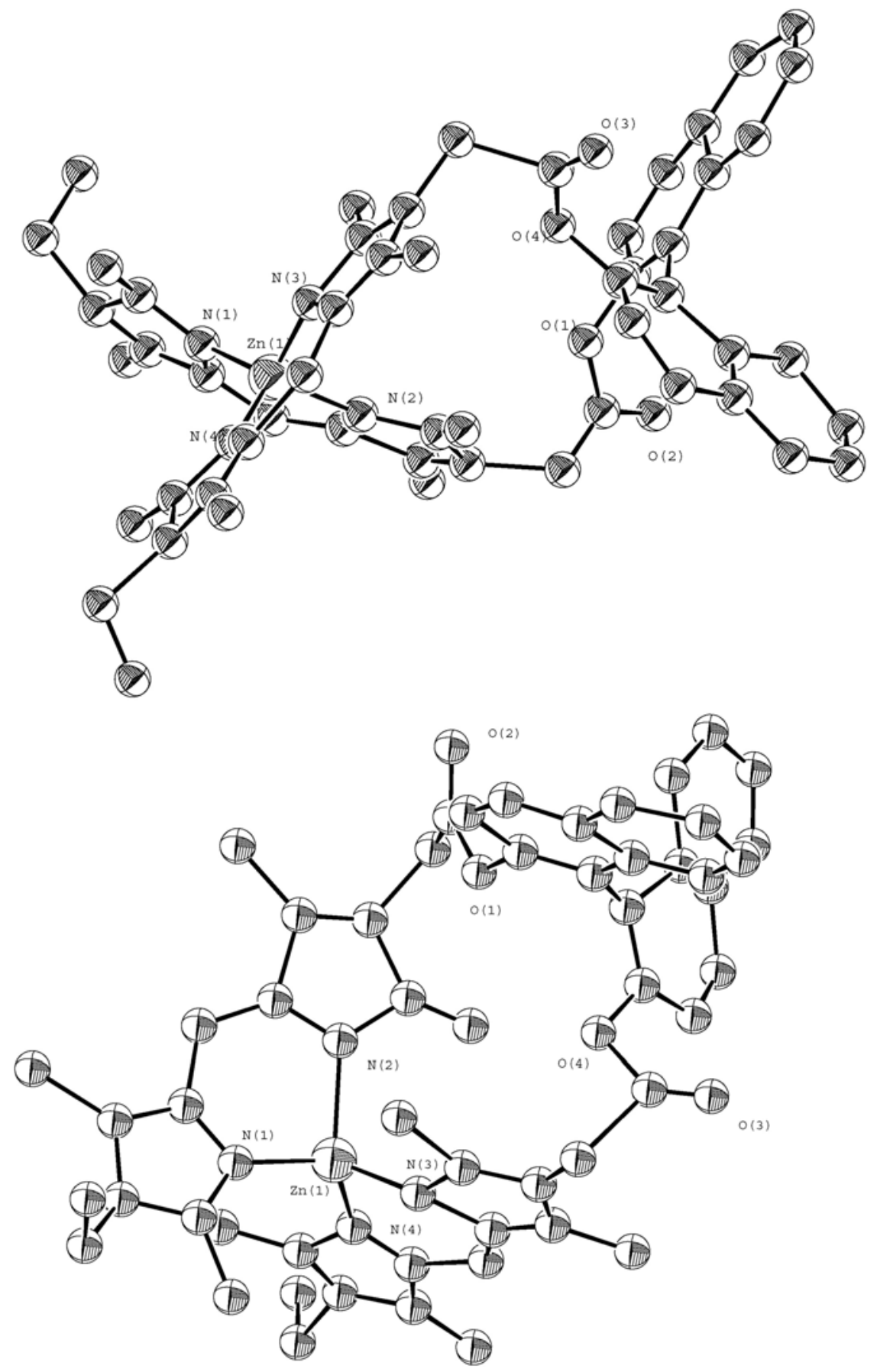

Unsatisfactory refinement was obtained (17\%).
a: $\quad 29.52(2)$
b: $\quad 15.87(1)$
c: $\quad 11.374(9)$

Orthorhombic

Space group: $\mathrm{P} 2{ }_{1} 2_{1} 2_{1}$ 
Highly diastereoselective templated complexation of dipyrromethenes

\section{CD spectra}

CD spectra for tartrate ligand 5 and zinc complex $7(d e=89 \%)$.

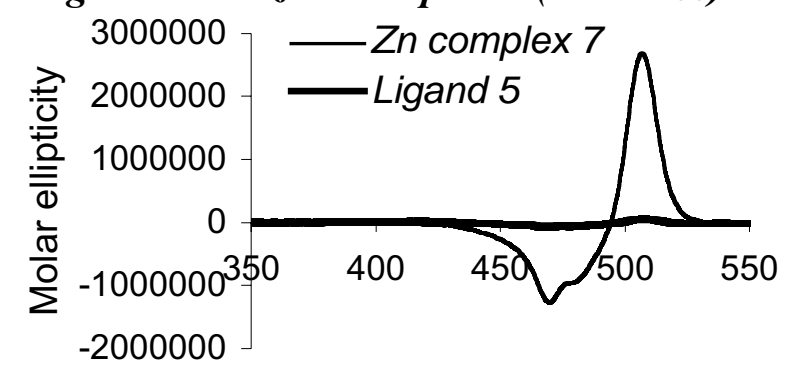

Wavelength/nm 
Highly diastereoselective templated complexation of dipyrromethenes

${ }^{13} \mathrm{C}$ NMR spectra

${ }^{13} \mathrm{C}$ spectrum of bis[benzyl 4,4'-[(R)-binolethanoate-3,5-dimethyl]-1H-pyrrole-2carboxylate]
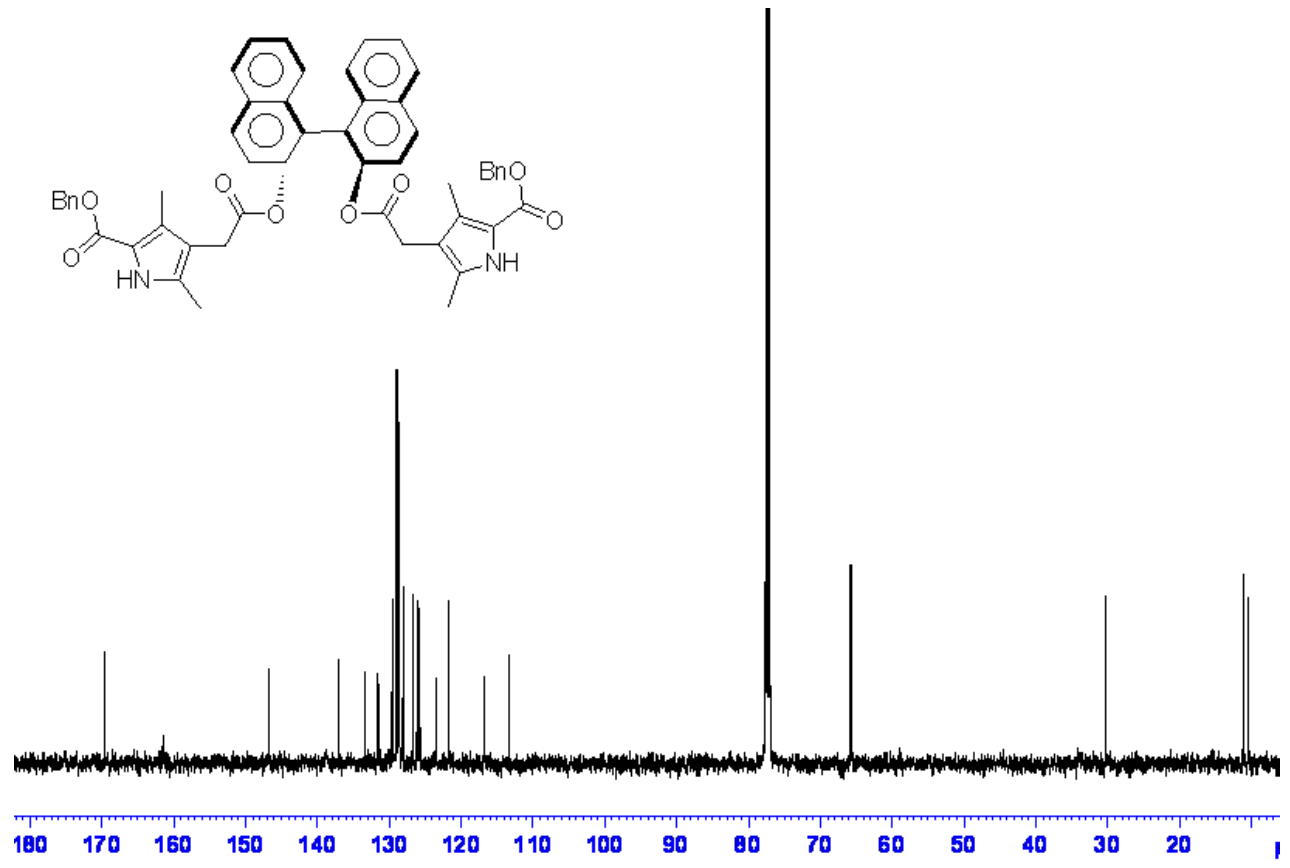

${ }^{13}$ C spectrum of 3,3'-[(R)-binolethanoate]bis[2,4,dimethyl-5-[(4-ethyl-3,5-dimethyl1H-pyrrol-2-ylidene)methyl]-, $1 \mathrm{H}$ pyrrole dihydrobromide (4)

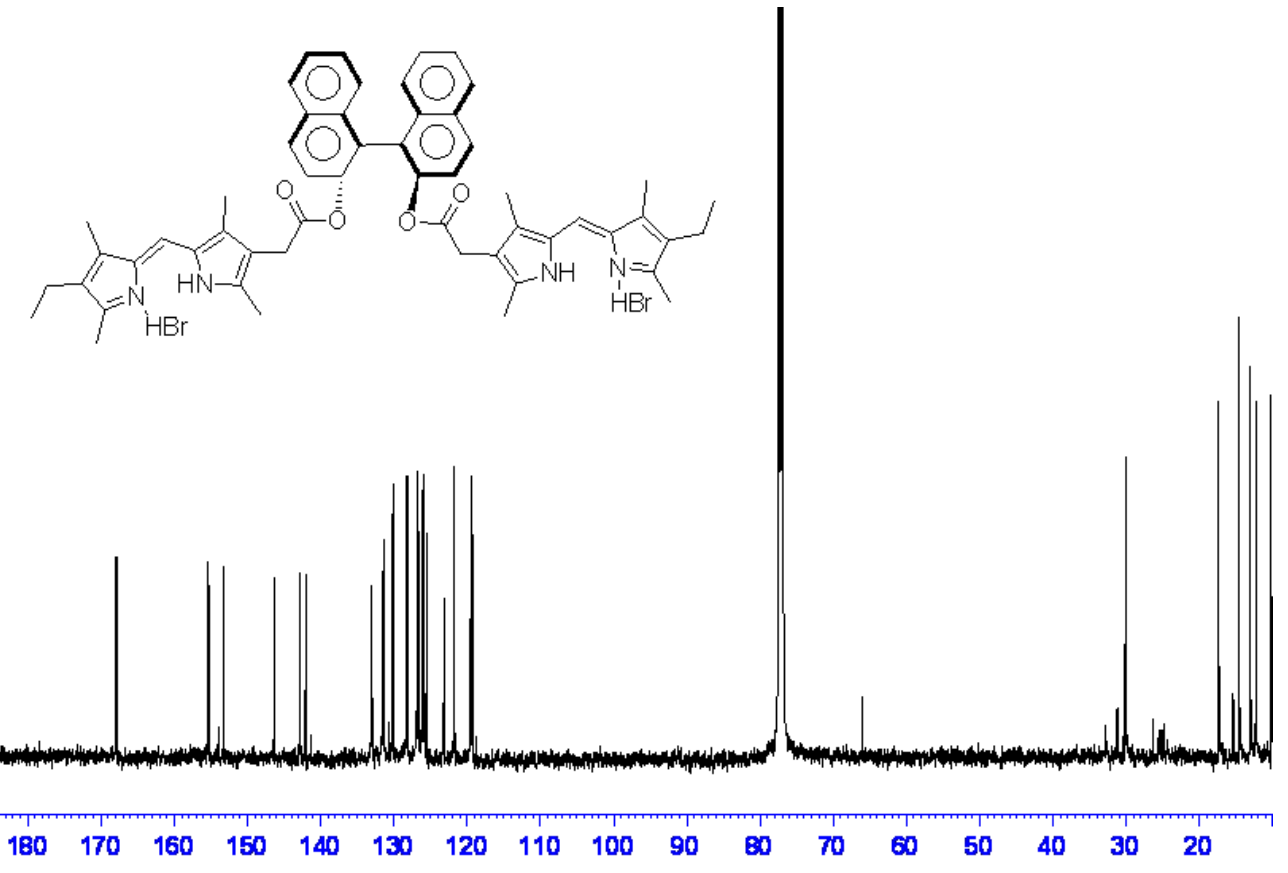


Highly diastereoselective templated complexation of dipyrromethenes

${ }^{13} \mathrm{C}$ spectrum of zinc, bis[m-[[3,3'-[(R)-binolethanoate $]$ bis [2,4,dimethyl-5-[(4-ethyl3,5-dimethyl-1H-pyrrol-2-ylidene)methyl]-1H-pyrrolato-kN]](2-)]]di- (6)
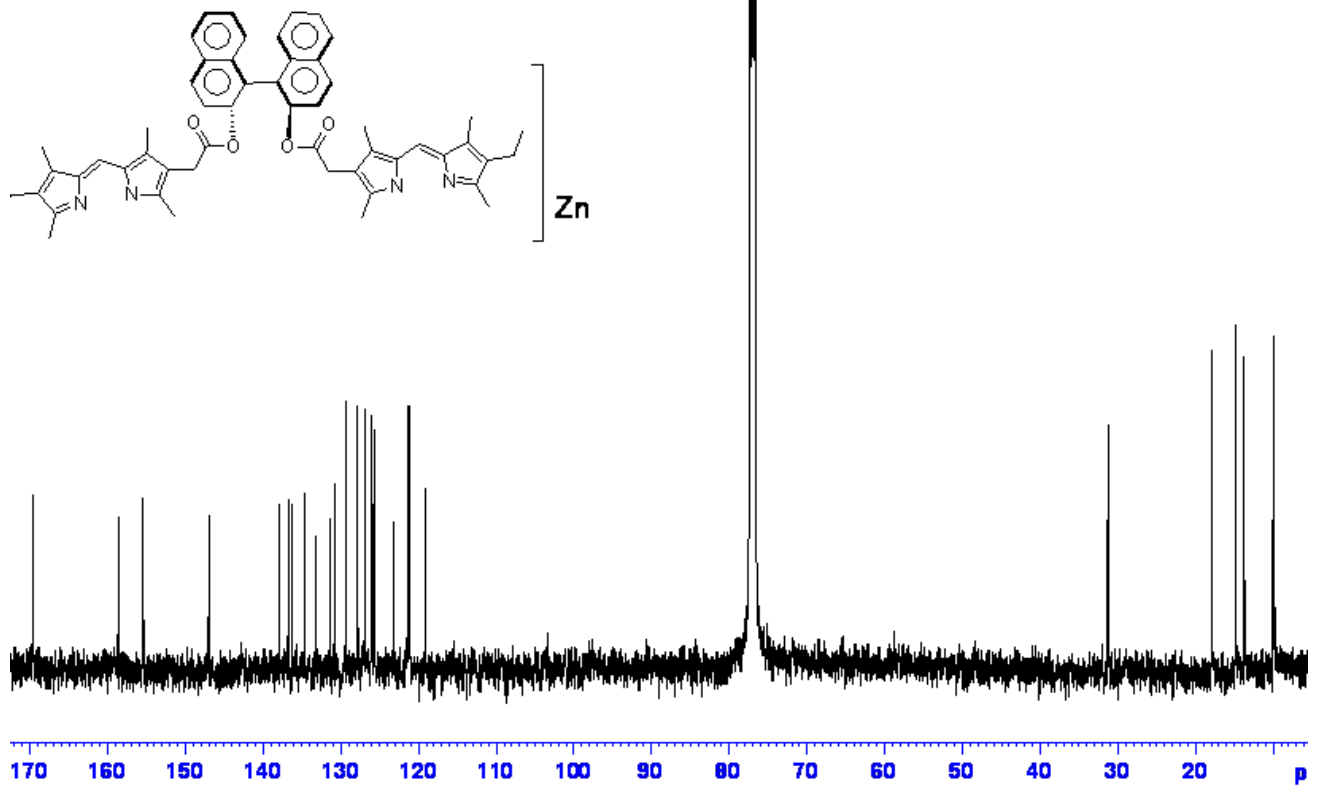

${ }^{13} \mathrm{C}$ spectrum of bis[benzyl 4,4'-[dimethyl-( $\left.R, R\right)$-tartrylethanoate-3,5-dimethyl]-1Hpyrrole-2-carboxylate]
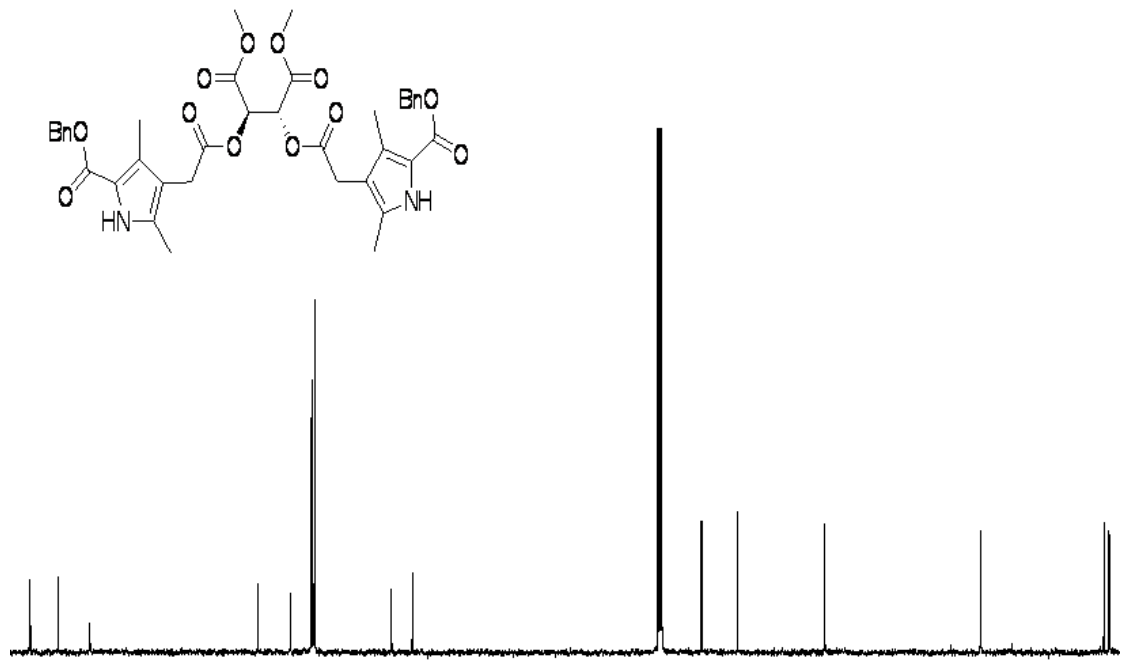

$\begin{array}{llllllllllllllll}170 & 160 & 150 & 140 & 130 & 120 & 110 & 100 & 90 & 80 & 70 & 60 & 50 & 40 & 30 & 20\end{array}$ 
Highly diastereoselective templated complexation of dipyrromethenes

${ }^{13} \mathrm{C}$ spectrum of 3,3'-[dimethyl-( $\left.R, R\right)$-tartrylethanoate]bis[2,4,dimethyl-5-[(4-ethyl3,5-dimethyl-1H-pyrrol-2-ylidene)methyl]-, $1 \mathrm{H}$ pyrrole dihydrobromide (5)
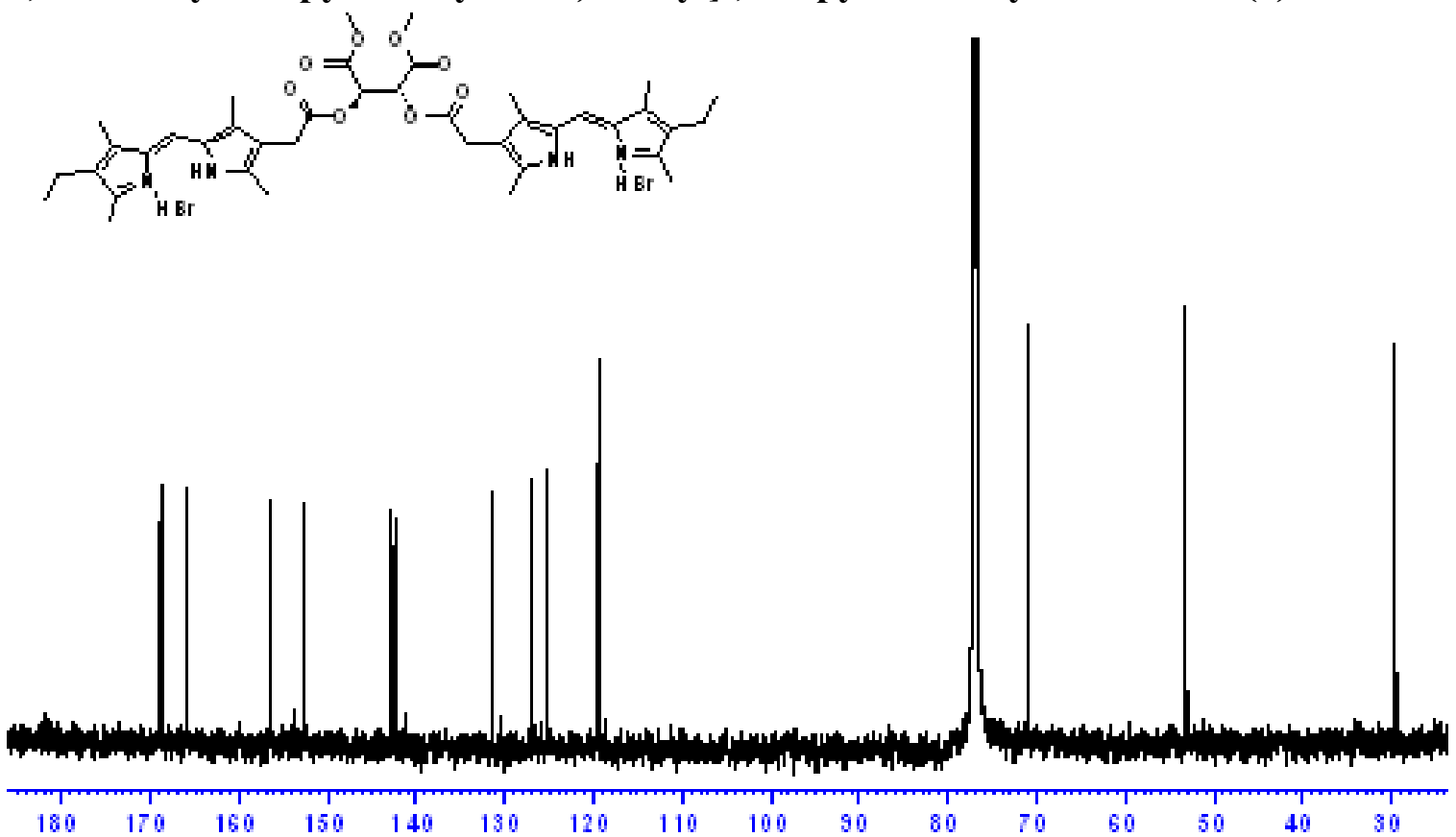

${ }^{13} \mathrm{C}$ spectrum of zinc, bis[m-[[3,3'-[dimethyl- $(R, R)-$ tartrylethanoate]bis [2,4,dimethyl-5-[(4-ethyl-3,5-dimethyl-1H-pyrrol-2ylidene)methyl]-1H-pyrrolato-kN]](2-)]]di- (7)
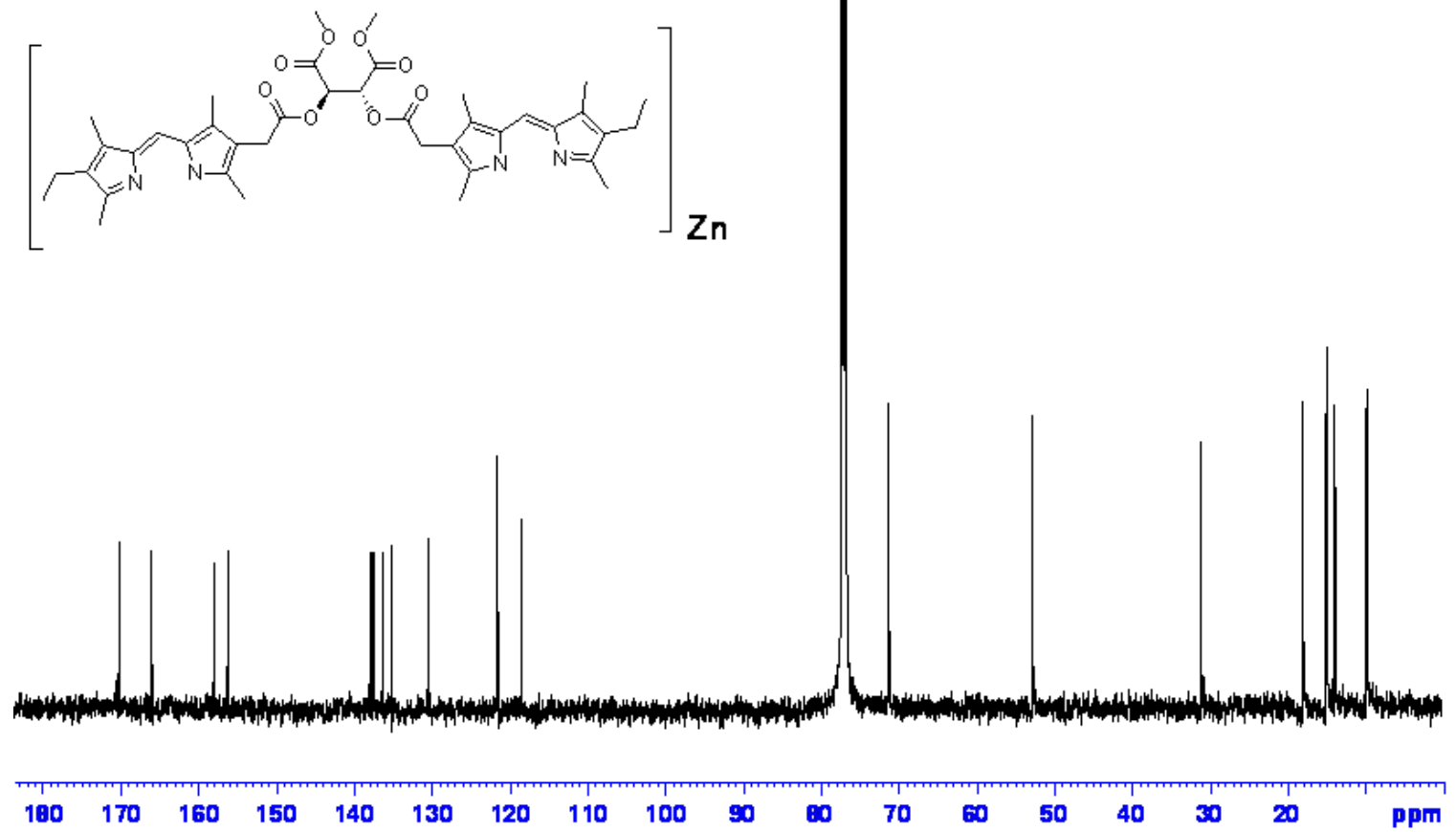